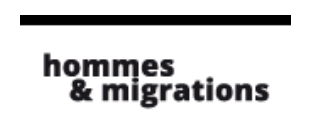

\section{Hommes \& migrations}

Revue française de référence sur les dynamiques

migratoires

$1319 \mid 2017$

Réfugiés et migrants au Liban

«Demeurer un musée de l'histoire de l'immigration et devenir un musée des migrants "

Entretien avec Benjamin Stora, président du Conseil d'orientation de l'EPPPD du Palais de la Porte Dorée-Musée national de l'histoire de l'immigration-Aquarium tropical

\title{
Marie Poinsot
}

\section{(2) OpenEdition}

Journals

Édition électronique

URL : http://journals.openedition.org/hommesmigrations/3955

DOI : 10.4000/hommesmigrations.3955

ISSN : 2262-3353

Éditeur

Musée national de l'histoire de l'immigration

Édition imprimée

Date de publication : 1 octobre 2017

Pagination : 6-8

ISBN : 978-2-919040-39-1

ISSN : $1142-852 X$

Référence électronique

Marie Poinsot, « «Demeurer un musée de l'histoire de l'immigration et devenir un musée des migrants » », Hommes \& migrations [En ligne], 1319 | 2017, mis en ligne le 01 octobre 2020, consulté le 06 janvier 2021. URL : http://journals.openedition.org/hommesmigrations/3955; DOI : https://doi.org/ 10.4000/hommesmigrations.3955 


\section{«DEMEURER UN MUSÉE DE L'HISTOIRE DE L'IMMIGRATION ET DEVENIR UN MUSÉE DES MIGRANTS »}

Entretien avec BENJAMIN STORA, président du Conseil d'orientation de l'EPPPD du Palais de la Porte Dorée-Musée national de l'histoire de l'immigration-Aquarium, réalisé par MARIE POINSOT.

\section{Hommes \& Migrations : \\ Dix ans après son ouverture, pouvez- vous nous rappeler les difficultés et le contexte tendu qui ont accompagné la préfiguration du Musée national de l'histoire de l'immigration et ses premières années?}

\section{Benjamin Stora :}

Il faut tout d'abord rappeler les polémiques qui ont accompagné la création du Musée national de l'histoire de l'immigration. Ce projet est né difficilement parce que son objet, l'histoire de l'immigration, est très sensible. Il y a d'abord eu plusieurs revendications de paternité. Au départ, ce projet repose sur une volonté politique et porte sur la manière d'écrire et de consolider dans la société française un récit historique faisant place à l'immigration au sein de l'histoire nationale. N’oublions pas le bouillonnement de projets, d'idées en faveur de la reconnaissance des mémoires qui a marqué le tournant des années 1990, dix ans après les mouvements dans les banlieues du type Vaulx-en-Velin ou la Marche pour l'égalité et contre le racisme. Ces propositions de lieux de mémoires ou autres se sont cristallisées avec l'accession du Front national au deuxième tour de lélection présidentielle de 2002. Elles se sont fédérées autour de la mission de préfiguration d'un Centre de ressources et de mémoires dont la présidence a été confiée par Jacques Chirac à Jacques Toubon et qui a abouti à l'ouverture de ce musée.

La deuxième difficulté est d'ordre méthodologique : ce projet devait-il être un simple lieu de mémoires ou, au contraire, une entreprise citoyenne? La réponse concrétisée à l'époque reste toujours valable à mon sens, à savoir que le Musée doit être les deux à la fois. Il s'agit de raconter une histoire de l'immigration et d'essayer de la faire vivre dans le présent, à travers tous les combats contre le racisme et pour la citoyenneté. Certains étaient opposés dans l'échiquier politique à cette idée de lieu de mémoires : selon eux, la France est une société homogène qui n'a pas besoin de disposer d'un tel lieu de mémoires puisqu'elle ne s'est pas construite sur l'immigration. On oublie trop souvent qu'une fraction politique de la société française a toujours été opposée à ce projet.

Lors de la mission de préfiguration du Musée, un autre débat a porté sur les limites du périmètre mémoriel. Le projet concernait-il tous ceux qui migrent ou se déplacent ? Devait-il aborder aussi la problématique des frontières, les rapports entre Français et étrangers, le dedans et le dehors? Au cœur de cette interrogation, bien sûr et surtout, il y avait la place de l'Algérie, dans sa relation avec l'histoire de France. Comment aborder l'histoire des Européens d'Algérie qui étaient à la fois citoyens 
français mais aussi des réfugiés dans l'ancienne métropole ? Fallait-il accorder une place particulière aux migrations post-coloniales dans l'histoire de l'immigration en France ? La question du périmètre se posait de même pour les Antillais et tous les ressortissants des territoires d'Outre-mer.

Lors de la mission de préfiguration, le débat s'est également emporté sur le choix du lieu lui-même, le Palais de la Porte Dorée, ce palais Art déco unique à Paris mais aussi le bâtiment phare de l'exposition coloniale internationale de 1931. Pourquoi situer un lieu de mémoires de l'immigration dans un bâtiment marqué par l'histoire coloniale française? Ma position sur ce sujet était qu'il ne fallait pas éluder l'histoire coloniale mais, au contraire, l'assumer comme une partie intégrante et structurante de l'histoire de l'immigration. En même temps, cette histoire complexe des relations entre colonisation et immigration se poursuit à travers l'histoire migratoire de manière plus globale.

Enfin, un autre débat très vif portait sur le soutien du ministère de l'Identité nationale qui était à l'époque le principal ministère de tutelle du projet et contre lequel se sont opposés certains historiens qui ont refusé d'apporter leur caution et leur légitimité scientifique à une entreprise qui semblait vouloir valoriser « l'identité nationale».

Voilà pourquoi il était si difficile de trouver un consensus sur la nature du lieu, sur les problématiques, sur les périmètres d'exploration méthodologique, sur le bâtiment lui-même, et sur le rapport à l'État. Ces raisons font que ce projet a été un musée à part, « devenant » difficile, d'autant plus qu'il n'a pas été inauguré par le président de la République en octobre 2007 ; ce qui a contribué à le marginaliser à la périphérie des entreprises muséales françaises.

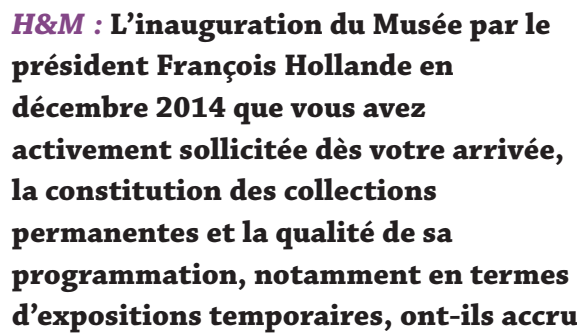

\section{sa légitimité auprès des pouvoirs publics ? Comment le Musée a-t-il acquis une reconnaissance auprès de l'État et des publics? Quel chemin reste-t-il encore à faire selon vous ?}

B. S. : Force est de constater que ce projet a très largement progressé dans ces domaines depuis 10 ans. À mon arrivée en 2014 comme président du Comité d'orientation, mes trois préoccupations étaient de convaincre le président de la République de la nécessité d'une inauguration officielle - qui fut réalisée par François Hollande en décembre 2014 -, d'obtenir des rallonges budgétaires pour l'entretien du bâtiment - le budget de l'établissement a été sensiblement augmenté -, de développer les activités dans toutes les disciplines artistiques et culturelles et les débats citoyens. Aujourd'hui, le Musée a acquis une visibilité dans les médias. Les expositions sont largement couvertes par la presse ; ce qui n'était pas le cas auparavant. Des thèses sur l'histoire de l'immigration et des films sont en préparation en partenariat avec le Musée.

Le travail de mise en œuvre muséale doit être poursuivi. Une des difficultés tient au fait que les immigrés eux-mêmes, qui sont les acteurs de cette histoire, ne souhaitent pas forcément qu'on la leur rappelle. Ils ont plutôt envie d'effacer les traces de leur arrivée et de leur installation en France. Mais c'est le propre de toutes les migrations. Or ce musée entend montrer ce que les populations concernées ou la société d'accueil veulent parfois effacer. Cela s'avère nécessaire pour fabriquer l'histoire de France en intégrant cette composante étrangère et en activant la transmission mémorielle d'une génération à l'autre, des populations immigrées vers le reste de la société française.

Par ailleurs, l'installation du Musée qui fut longue et le retard pris en termes de reconnaissance et de visibilité dans l'espace public s'inscrivent dans un contexte où l'extrême droite française, qui rejette totalement toute l'histoire liée à l'immigration, est montée en puissance. Dans le fond, ce musée est confronté à une course de vitesse implacable pour contrer cette idéologie nationaliste. C'est pourquoi un musée qui porte sur cette histoire particulière 
que l'on veut enfouir est devenu aujourd'hui une nécessité absolue.

\section{H\&M : Le Musée national de l'histoire de l'immigration a pour mission de contribuer à la diffusion des savoirs sur l'immigration dans la société française. Comment percevez-vous l'évolution des travaux sur la question migratoire ? Le domaine des études migratoires est-il en train de se fondre dans l'ensemble des sciences humaines et sociales?}

B. S. : Le dynamisme de ce musée repose effectivement sur l'intérêt et l'implication des nouvelles générations d'intellectuels et de chercheurs sur les questions coloniales et migratoires. Nous avons récemment analysé le développement des recherches sur ces deux grands thèmes dans le rapport remis aux ministères de la Culture et de la Recherche en mars 2017, intitulé : "La recherche sur les migrations et l'immigration. Un état des lieux ». Cette génération de chercheurs montre un intérêt très fort pour la façon dont la société française s'est fabriquée à partir des migrations d'hier et d'aujourd'hui. Ainsi, il s'agit de dépasser le discours sur l'homogénéité nationale, jacobine, républicaine et de solliciter d'autres récits et de diffuser d'autres savoirs pour mieux comprendre de tels processus de construction de la société. Face aux débats actuels, parfois violents et caricaturaux sur les migrations, sur la citoyenneté, sur le modèle de société, le Musée joue son rôle de sélection et de diffusion des connaissances. Il n'offre pas seulement une plongée dans un passé rassurant mais il permet de comprendre les troubles de la société d'aujourd'hui. Il s'agit pour ce musée d'être un véritable passeur de savoirs.

Ce faisant, le Musée doit prendre en compte la révolution numérique dans la circulation des idées, identifier les nouveaux acteurs sur les territoires, et les nouveaux médias qui sont adaptés et utilisés par les publics, les jeunes notamment, pour servir de relais du Musée dans les régions et les villes. Le Musée doit être capable d'évoluer et de modifier de manière considérable sa façon d'écrire, de penser et de diffuser l'histoire de l'immigration.

Le drame des migrants en Méditerranée vient chaque jour nous rappeler que la question migratoire s'est élargie à l'Europe et à l'échelle mondiale. Il ne s'agit plus de l'immigration classique, de recrutement économique ou de regroupement familial que la France a connu dans les années 1980. Aujourd'hui, ce qu'on appelle la "question » des migrants s'est renouvelée et interroge les catégories des migrations qui ont été forgées par le passé. L'accélération et la mondialisation des flux migratoires provoquent un basculement des cadres de pensée établis sur les migrations et les modèles d'exil qui se déploient dans une situation d'urgence obligent les États comme les musées à concevoir différemment ces problématiques migratoires. Peut-être s'agit-il à présent d'élargir notre périmètre muséal en posant une nouvelle hypothèse : comment demeurer un musée de l'histoire de l'immigration et devenir un musée des migrants dans leur singularité et leur diversité ? À la rentrée, la programmation culturelle intitulée Welcome sera ainsi consacrée aux situations des réfugiés et, à l'avenir, le Musée souhaite pouvoir la renouveler chaque année en y intégrant les thématiques d'actualité. 